\title{
An Evaluation on Transfection Efficiency of pHRE-Egr 1-EGFP in Hepatocellular Carcinoma Cells Bel-7402 Mediated by PEI-MZF-NPs
}

\author{
Mei Lin, ${ }^{1,2}$ Dongsheng Zhang, ${ }^{1}$ Junxing Huang, ${ }^{2}$ Jia Zhang, ${ }^{1}$ Li Wang, ${ }^{2}$ Ting Guo, ${ }^{2}$ \\ Li Xiao, ${ }^{2}$ Jun Ye, ${ }^{2}$ and Lixin Zhang ${ }^{2}$ \\ ${ }^{1}$ Medical School of Southeast University, Jiangsu Province, Nanjing 210009, China \\ ${ }^{2}$ Taizhou People's Hospital, Yangzhou University, Jiangsu Province, Taizhou 225300, China \\ Correspondence should be addressed to Dongsheng Zhang, zdszds1222@163.com
}

Received 12 June 2011; Accepted 20 June 2011

Academic Editor: Daxiang Cui

Copyright () 2011 Mei Lin et al. This is an open access article distributed under the Creative Commons Attribution License, which permits unrestricted use, distribution, and reproduction in any medium, provided the original work is properly cited.

To improve transfection and expression efficiency of target gene, especially under cancer anoxic microenvironment, we have developed pHRE-Egr1-EGFP/PEI-MZF-NPs nanosystem, in which pHRE-Egr1-EGFP, eukaryotic gene expression plasmid, is constructed by combining radiation promoter Egrl with anoxia induction components (HRE), forming anoxic radiation double sensitive HRE/Egr1 promoter to activate reporter gene EGFP expression. MZF-NPs $\left(\mathrm{Mn}_{0.5} \mathrm{Zn}_{0.5} \mathrm{Fe}_{2} \mathrm{O}_{4}\right.$ magnetic nanoparticles), obtained by coprecipitation method, are coated with cation poly(ethylenimine) (PEI). We transferred pHRE-Egr1-EGFP into hepatocellular carcinoma Bel-7402 cells, using PEI-MZF-NPs as the carrier and tested some relevant efficacy. The results show that PEI-MZF-NPs have good DNA-binding ability, protection ability, release ability, little toxicity, and high transfection efficiency, obviously superior to those of the liposome method and electricity perforation method. Moreover, the expression level of EGFP gene induced by anoxia and radiation was significantly higher than that of single radiation activation. It is therefore concluded that HRE/Egrl can induce and improve target gene expression efficiency in cancer anoxic microenvironment, and that PEI-MZF-NPs can be used as a novel nonviral gene vector which offers a viable approach to the mediated radiation gene therapy of cancer.

\section{Introduction}

Developed recently to address the problems in gene therapy and radiation therapy, radiation-gene therapy is a promising area of study in cancer treatment. More specifically, it is a cancer therapeutic method that while doing local radiotherapy, the genes with antitumor effect are induced by the radiation to express to kill cancer cells, resulting in dual therapy of ray and gene on cancer, thus working together to their best advantage, and playing a synergistic antitumor role. Egr1, a transcription factor, regulating cell growth early after being radiated, can induce the expression of its downstream genes by ionizing radiation, attaining a spatiotemporal regulation on the target gene expression. Notable achievements in radiation-gene therapy by using radiosensitivity of Egr1 radiation promoter have been obtained [1$3]$. However, this method has not overcome the common problem in gene therapy yet, that is, how to ensure a safe gene delivery into cells with high transfection efficacy. In addition, the anoxic microenvironment in solid tumor is likely to curb the induction activity of radiation promoter.

Hypoxia response element (HRE), an enhancer sensitive to hypoxia, can induce the expression of its downstream genes when combined particularly with HIF-1, hypoxia inducible factor-1. It was reported that HRE/HIF system existed in both mammalian cells and human tissue, and HIF$1 \alpha$ was overexpressed in $68-84 \%$ of cancers, which suggested that HRE can be used to regulate expression of target gene in tumor hypoxia environment [4-7]. Shibata et al. [8] has confirmed that 5 copies of HRE connected with a promoter can increase its downstream gene expression by 500 times in hypoxia condition.

In this study, 5HRE was inserted in the front of Egr1 promoter to construct a hypoxia-radiation dual-sensitive 
promoter and then connected with EGFP, a reporter gene, to develop an eukaryotic recombinant plasmid-pHRE-Egr1EGFP to induce and improve EGFP expression in tumor cells using hypoxia and radiation. In this system, radiation can be used as a switch to start the transcription of target gene, regulating its expression in space-time. Because of the radiation dose, range and times can be artificially controlled; the expression of the target gene is likely to occur within a certain range, at an appropriate time, even at the desirable level. Thus, the goal of controlling gene expression can be achieved to some extent, and the level of target gene expression will be improved in hypoxic microenvironment of solid tumors.

The choice of gene transfer vector is another key issue in gene therapy. As two major kinds of gene transfer vectors currently viral vector system and nonviral vector system both have their own advantages and disadvantages. Being the most efficient so far, the former is not regularly used in clinic due to small gene capacity, poor targeting specificity, selfimmunogenicity, and serious biosafety risk in particular. In spite of having avoided the major security risk, the latter is greatly inferior to the former in transfection efficiency, and meaningful expression of target gene is hardly available. Currently lipofection and electroporation are the two nonviral vector transfection methods used most. But besides high cytotoxicity, liposome can be quickly cleared by serum in vivo, which greatly limits its utility, in spite of good transfection efficiency [3]. Electroporation method with high efficiency is suitable for transient expression and stable transfection, but it only applies to cells or tissue in vitro, not suitable for transfection in vivo. Moreover, a large number of cells will be killed by electric shocks [9-11].

Encouragingly, nanotechnology developed recently has offered a new means for solving the problem of gene transfer vector [12-19]. The research on the gene transfer vector based on nanoparticle has attracted wide attentionthe therapy genes such as DNA or RNA wrapped in the nanoparticles or adsorbed on the surface are taken into cells and released. Compared with traditional carriers, nanovectors used for gene transfer have a lot of advantages such as nonimmunogenicity, repeated injection, no genetic toxicity and cytotoxicity, no cell transformation or death, slow release of the genes to extend response time effectively, and maintaining effective concentration of the product, improving transfection efficiency and bioavailability of the product, and so on $[20,21]$. Such vectors have become a new promising carrier system because they not only integrate advantages of the virus vectors and traditional non-viral vectors, but also avoid their defects [22-28]. Particularly, besides properties of general nanoparticles, the superparamagnetic effect of magnetic nanoparticle gene transfer vector will produce transfection with high efficiency and directional movement in an external magnetic field, which in turn helps to carry out targeted gene therapy.

But it is well known that the smaller particles, the more agglomerate, and soft agglomeration widely exist in nanoparticles (especially magnetic nanoparticles). Only modified on the surface can nanoparticles get into the cells to play a role of gene transfer vector, and dispersion of

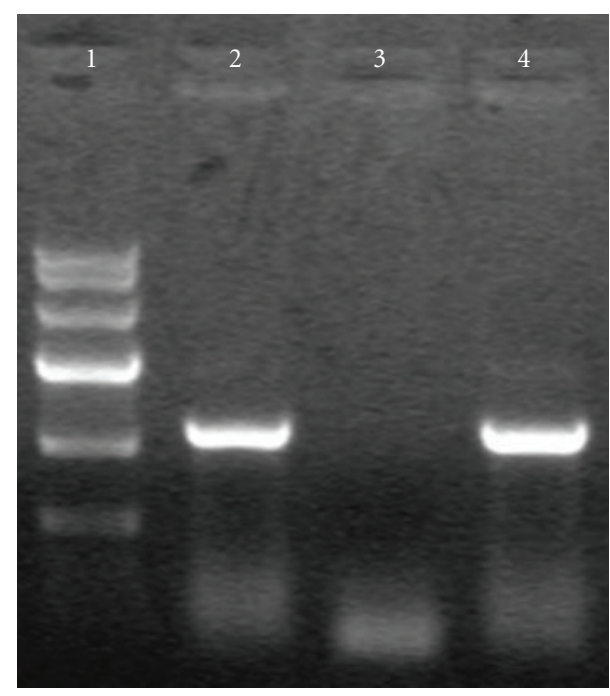

FIGURE 1: PCR gel electrophoresis of pCDNA3.1-Egr1-EGFP transfected (lane 1: Marker IV (each band in turn from top to bottom is $7 \mathrm{~K}, 5.5 \mathrm{~K}, 3.5 \mathrm{~K}, 2 \mathrm{~K}, 1 \mathrm{~K}, 500 \mathrm{bp}$ ); lane 2: pCDNA3.1-Egr1-EGFP; lane 4: pCDNA3.1-Egr1-EGFP).

the particles and surface functional groups have a direct impact on the further application of particles $[13,17]$. As a common powder surface modification agent, PEI, whose monomer (-CH - $\mathrm{CH} 2-\mathrm{NH} 2-)$ has good ability to bind DNA and adhere to cells, has electrostatic repulsion and steric hindrance effect [29-31]. In the previous study in our lab, $\mathrm{Mn}_{0.5} \mathrm{Zn}_{0.5} \mathrm{Fe}_{2} \mathrm{O}_{4}$, a thermosensitive $\mathrm{Mn}$, and $\mathrm{Zn}$ ferrite magnetic nanoparticles, were successfully prepared chemically and modified surfacely by PEI, and good transgenic effect on cos-7 cells was obtained [32]. But whether PEIMZF-NPs can be used for transfection of pHRE-Egr1-EGFP into cancer cells to perfect radiation-gene therapy for tumors and what the effect will be remain unknown to us.

In this project, attempts were made to develop a pHREEgr1-EGFP/PEI-MZF-NPs composite nanosystem, in which pHRE-Egr1-EGFP were transferred into hepatocellular carcinoma Bel-7402 cells by PEI-MZF-NPs as the carrier, and some relevant efficacy was tested. In addition, a comparative study of the level of gene expression by double induction of hypoxia and radiation and separate induction of radiation was made, which was expected to offer a viable approach to the mediated radiation gene therapy of cancer.

\section{Materials and Methods}

2.1. Main Reagent. Lipofectamine TM2000 purchased from Invitrogen Corporation; PEI (polyethylenimine, PEI) and dimethyl sulfoxide (DMSO) purchased from Sigma; Agarose purchased from MRI; DNaseI from Amercso; DMEM medium and fetal bovine serum from Gibco; Thiazolyl blue (MTT) from AMRESCO; PCDNA3.1-EGFP from Biotech Co.Ltd. Changsha Ying Run. Bel-7402 cells were provided by the Institute of Biochemistry and Cell Biology, Shanghai Institute of Biological Sciences, Chinese Academy of Sciences. 


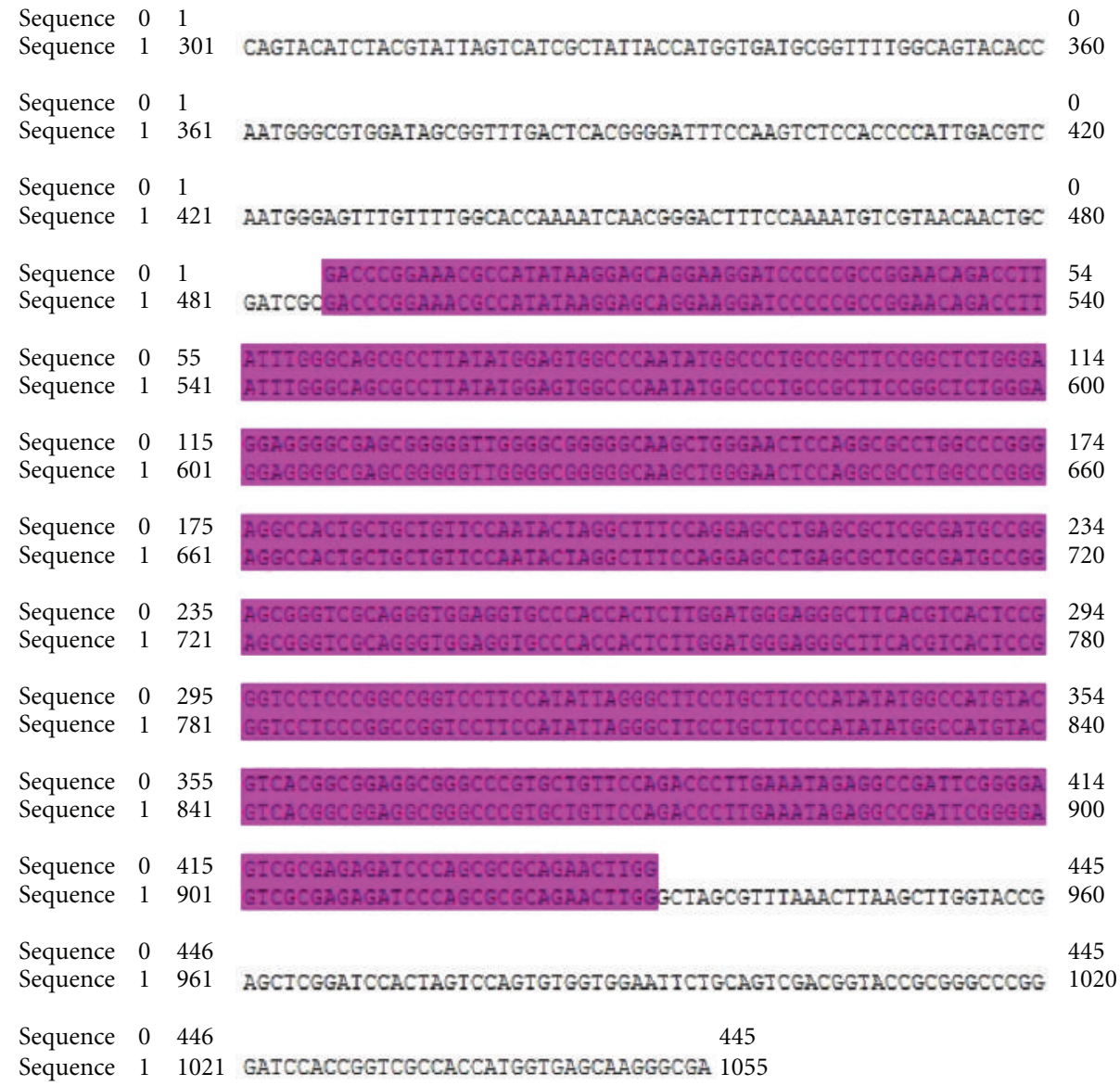

Figure 2: Sequencing and alignment of pCDNA3.1-Egr1-EGFP (sequence 0: Egr1 promoter sequences without CMVE; sequence 1: pCDNA3.1-Egr1-EGFP sequences with ab1 reverse complementary sequences; software: Dnassit 2.0).

\subsection{Construction and Identification of the Eukaryotic} Expression Plasmids: Egr1-HRE-EGFP

2.2.1. PCR Amplification of Egr1 Promoter Segments. Egr1 promoter was inserted into pIRES vector. In front of the Egr1 promoter, there was a CMV fragment to enhance the expression efficiency of the promoter. The primer sequences were designed according to CMVE-Egrlp template, and CMVE-Egrlp fragments were amplified by PCR.

Egr1-f: 5'-CCG CTCGAG CG ACGCGT GATCTTCAATATTGGCCATTAGC-3'

-MluI: CG ACGCGT;

-XhoI: CCG CTCGAG

Egr1-r: 5'-CCG CTCGAG CTA GCTAGC CCAAGTTCTGCGCGCTGGGAT-3'

-NheI: CTA GCTAGC

-XhoI: CCG CTCGAG
2.2.2. Egr1p Were Subcloned into pCDNA3.1-EGFP. The above CMVE-Egr1p fragments amplified were collected and subcloned into pCDNA3.1-EGFP on MluI and NheI sites. After transfection, the plasmids were purified and identified by PCR.

Egr1-f: 5'-CCG CTCGAG CG ACGCGT GATCTTCAATATTGGCCATTAGC-3',

Egr1-r: 5' -CCG CTCGAG CTA GCTAGC CCAAGTTCTGCGCGCTGGGAT-3'.

Product length $=1152 \mathrm{bp}$.

According to the PCR electrophoresis results, the correct pCDNA3.1-Egr1-EGFP sequences were selected to test, EGFP-N-3 as the sequence primer.

2.2.3. To Synthesize 5HRE. 5HRE was synthesized according to [33]. In order to facilitate subsequent experiment, several restriction sites were added on the two sides of 5HRE. Synthetic products were identified by enzyme cleavage and gene sequence analysis (gene name: 5HRE; gene length: 185 bp; carrier name: pUC57; cloning site: EcoRI, HindIII).

2.2.4. Construction of pcDNA 3.1-5HRE-Egr1p-EGFP. PUC57-5HRE and pCDNA3.1-Egr1p-EGFP were connected

Product length $=1152 \mathrm{bp}$. 

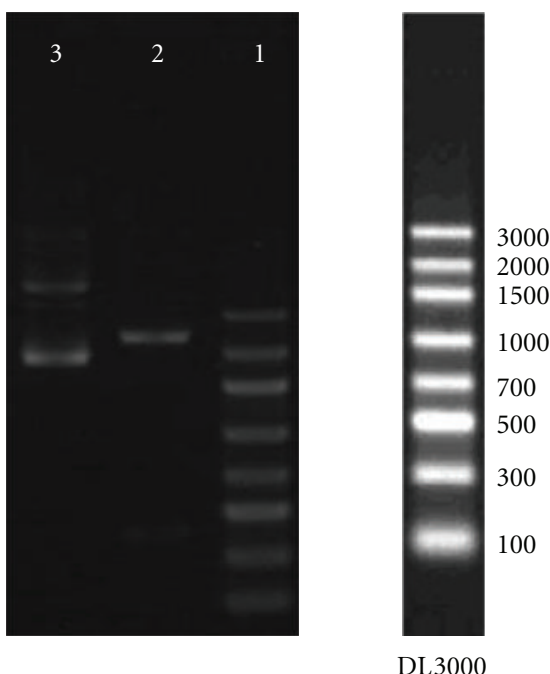

Figure 3: pUC57-5HRE was identified by restriction enzyme digestion (Lane 1: DL3000; lane 2: pUC57-5HRE digested by NdeI and HindIII; lane 3: pUC57-5HRE).

after digestion by MluI, respectively. Then the connected products were transfected into DH5 $\alpha$. At last, the plasmids were isolated and purified after enlargement culturing and verified by BglII enzyme cleavage and gene sequence analysis.

\subsection{MZF-NPs Preparation and Modification}

2.3.1. Preparation. MZF-NPs were obtained by Chemical Coprecipitation Method, referring to [34].

2.3.2. Surface Modification [35]. Firstly, some MZF-NPs were dissolved in deionized water to prepare $4 \%$ of the magnetic fluid; supernatant was discarded after ultrasonic dispersion and high-speed centrifugation. Secondly, the precipitate was resuspended in PBS and ultrasonic dispersed. Thirdly, PEI was added slowly to the resuspender. The mixture was blended in a shaker at constant temperature for $24 \mathrm{~h}$. Magnetic particles were separated from the solution by magnetic method and washed repeatedly by distilled water and methanol. After being vacuum dried, nanomagnetic particles modified surfacely by PEI were obtained (namely PEI-MZF-NPs).

2.4. Binding Assay of PEI-MZF-NPs and pHRE-Egr1-EGFP. PEI-MZF-NPs and pHRE-Egr1-EGFP were mixed together at mass ratios $0: 1,5: 1,10: 1,20: 1,40: 1$, and $80: 1$ (the final volume was $500 \mu \mathrm{L}$, and DNA concentration was constantly $0.01 \mu \mathrm{g} / \mu \mathrm{L}$ ). 30 minutes later at room temperature, $10 \mu \mathrm{L}$ of the above composite was taken, respectively, to test DNA binding ability of magnetic nanoparticles using agarose gel electrophoresis. According to the electrophoresis results, the optimal ratio of binging DNA with nano-magnetic particles could be observed.
2.5. DNase-I Digestion Experiment of PEI-MZF-NPs-DNA. PHRE-Egr1-EGFP and PEI-MZF-NPs were mixed in Tris Buffer, containing $60 \mathrm{mM} \mathrm{MgCl}_{2}$, at 1:40 of mass ratio. 30 minutes later at room temperature, DNase I was added to the above mixture, water bathing at $37^{\circ} \mathrm{C}$. Stop solution ( $400 \mathrm{mmol} / \mathrm{L} \mathrm{NaCI}, 100 \mathrm{mmol} / \mathrm{L}$ EDTA) was used to terminate the reaction at 10,20, $40 \mathrm{~min}$ of digestion. pHREEgr1-EGFP was eluted from the composite by SDS, then extracted by phenol and chloroform, precipitated by absolute ethyl alcohol, and washed by $75 \%$ ethanol. The sediment was dissolved in distilled water, and the product was taken to do agarose gel electrophoresis assay to observe the ability of pHRE-Egr1-EGFP/PEI-MZF-NPs to resist nucleic acid enzymatic hydrolysis. As a control, the naked plasmids pHRE-Egr1-EGFP were done by the same approach.

2.6. DNA Release Assay for DNA-PEI-MZF-NPs. pHREEgr1-EGFP and PEI-MZF-NPs were mixed at 1:40 of mass proportion to form a composite. Then the composite was stirred in a shaker at $37^{\circ} \mathrm{C}$ after being dissolved by TE. Respectively, at $1 \mathrm{~h}, 4 \mathrm{~h}, 8 \mathrm{~h}, 12 \mathrm{~h}, 1 \mathrm{~d}, 2 \mathrm{~d}, 3 \mathrm{~d}, 4 \mathrm{~d}$, the solution was centrifuged at $15000 \mathrm{r} / \mathrm{min}$, and then $20 \mu \mathrm{L}$ supernate was taken to do agarose gel electrophoresis to observe DNA release ability of PEI-MZF-NPs-DNA. TE was replenished whenever some supernatant was taken, and the stir was continued.

\subsection{To Evaluate Transfection Efficiency of PEI-MZF-NPs and the Gene Expression Induced by Anoxia and Radiation}

2.7.1. Cell Culture. Bel-7402 cells were cultured in DMEM medium supplemented with $10 \%$ heat-inactivated calf serum, penicillin ( 100 units $/ \mathrm{mL})$, and streptomycin $(0.1 \mathrm{~g} /$ liter) and grew in the presence of $5 \% \mathrm{CO}_{2}$ in air at $37^{\circ} \mathrm{C}$.

2.7.2. Evaluation of Transfection Efficiency and Gene Expression Induced by Anoxia and Radiation. pHRE-Egr1-EGFP were transfected into Bel-7402 cells by using PEI-MZF-NPs as carrier, and the transfection efficiency was evaluated, compared with that of lipofection transfection method and that of electroporation transfection method.

PEI-MZF-NPs transfection: (1) the plasmid DNA and PEI-MZF-NPs were diluted with serum-free culture medium, respectively, and then they were mixed together (mass ratio of PEI-MZF-NPs and DNA was 40:1). 30 minutes later at room temperature, pHRE-Egr1-EGFP/PEIMZF-NPs composite was obtained. (2) The Bel-7402 cells were seeded in 6-well plates $\left(5 \times 10^{5}\right.$ cells per well $)$ and incubated in routine conditions. About 18 hours later $(80 \%$ cells were confluent), the original culture medium was discarded, and the cells were washed twice by PBS and washed once by DMEM with no serum. (3) Serum-free DMEM with pHRE-Egr1-EGFP/PEI-MZF-NPs was added to the wells (Every well contained $3 \mu \mathrm{g}$ DNA), and then the plates continued to be in the couveuse. 5 hours later, the medium with no serum was replaced by fresh DMEM medium with serum, and then the cells continued to be incubated for $48 \mathrm{~h}$. 


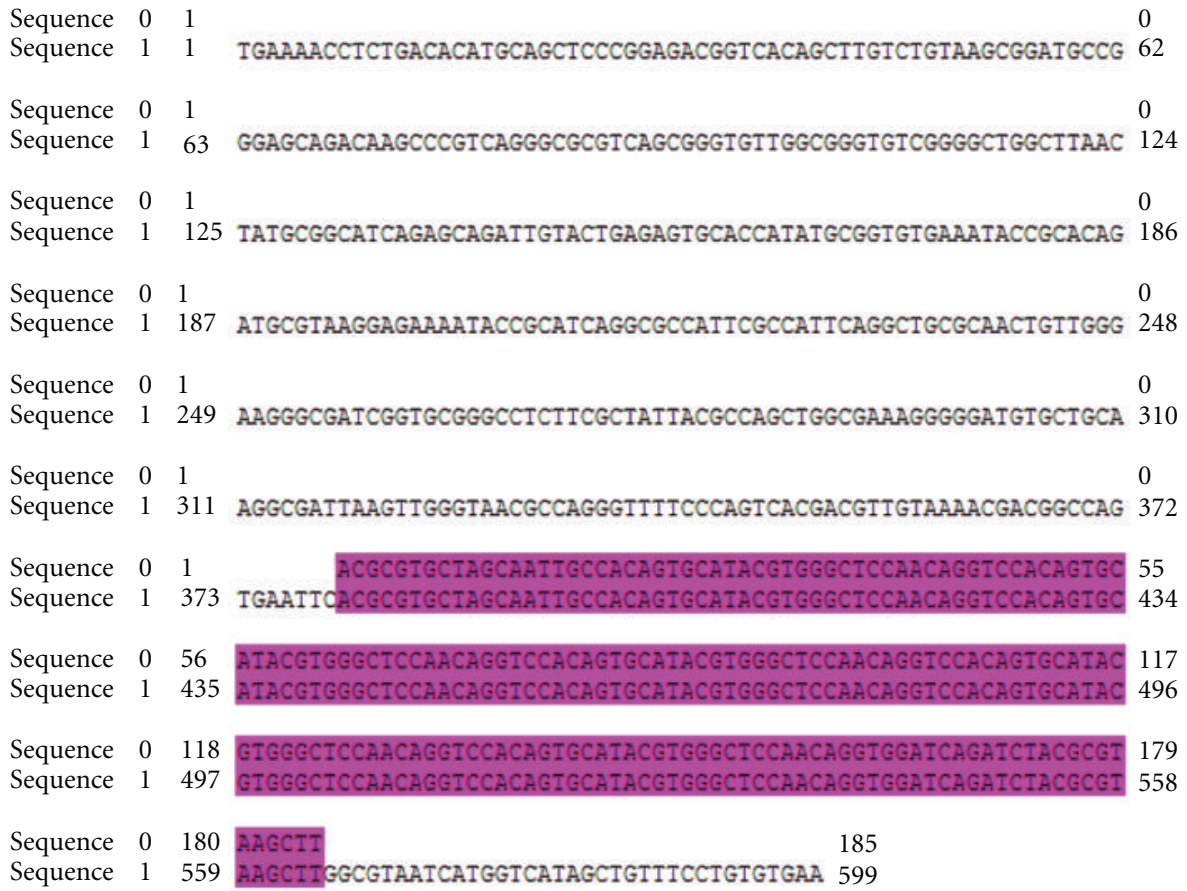

FIGURE 4: Sequencing and alignment of pUC57-5HRE (sequence 0: 5HRE; sequence 1: 5HRE with ab1 reverse complementary sequences; software: Dnassit 2.0).
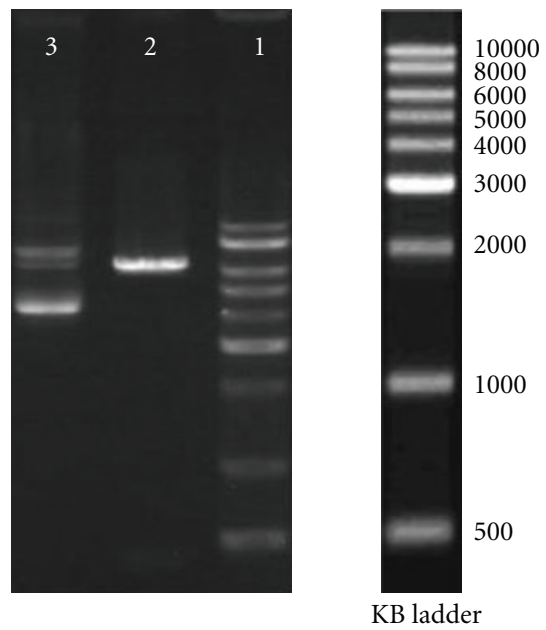

Figure 5: PCDNA3.1-5HRE-Egr1-EGFP was identified by restriction enzyme digestion (lane 1: KB Ladder; lane 2: pCDNA3.15HRE-Egr1p-EGFP digested by BglII; lane 3: pCDNA3.1-5HREEgrlp-EGFP).

Liposome method: (1) the plasmid DNA and liposome were diluted with serum-free culture medium, respectively, and then they were mixed together (mass ratio of PEIMZF-NPs and DNA was 40:1). 30 minutes later at room temperature, DNA-liposome composite was obtained. (2) The Bel-7402 cells before transfection were done by the same way as PEI-MZF-NPs group. (3) Serum-free DMEM with DNA-liposome was added to the wells (every well contained $3 \mu \mathrm{g}$ DNA, mass ratio of liposome and DNA was $5: 3$ ). After plates continued to be incubated for 5 hours, the medium with no serum was replaced by fresh DMEM medium with serum, and then the cells continued to be incubated for $48 \mathrm{~h}$.

Electroporation transfection: (1) the cells, digested by trypsin, were collected and centrifuged for 5 minutes $(1000 \mathrm{~g})$ after the trypsin reaction was terminated with culture medium with serum. (2) After the supernatant was discarded, the cells were washed twice by $1.25 \% \mathrm{E}$ buffer (containing $1.25 \%$ DMSO in medium with no serum). (3) The cells were diluted with concentration of $5 \times 10^{6} / \mathrm{mL}$ by E buffer. (4) $3 \mu \mathrm{g}$ DNA was added to every $400 \mathrm{~mL}$ of the cell suspension. (5) When being blended, the above mixture was transferred to electrode cup. (6) Electrode parameters were $250 \mathrm{v} / 950 \mathrm{uf}$, and the thickness of the electrode cup was $4 \mathrm{~mm}$. (7) The electrode cup containing cells and DNA was placed in the electric box and received the electric shock. Then it was kept at room temperature for 10 minutes, and the cell suspension was transferred into full culture medium and then cultured in routine conditions. $24 \mathrm{~h}$ later, fresh full DMEM medium was used to replace the old medium to abandon dead cells, and then the cells continued to be incubated for $48 \mathrm{~h}$.

The cells, transfected by the above three methods, were exposed to X-ray at $4 \mathrm{~Gy}(6 \mathrm{Mev})$ and then cultured for $24 \mathrm{~h}$ at $37^{\circ} \mathrm{C}$ in hypoxia environment $\left(0.1 \% \mathrm{O}_{2}, 5 \% \mathrm{CO}_{2}\right.$ and $\mathrm{N}_{2}$ balance gas). Fluorescent protein expressed in the cells was observed under the fluorescence microscope, and fluorescence intensity and transfection ratio were tested quantitatively by flow cytometric analysis. As a control, a group transfected by PEI-PEI-MZF-NPs and induced by radiation alone was designed, and the untransfected cells were used as a negative control. 


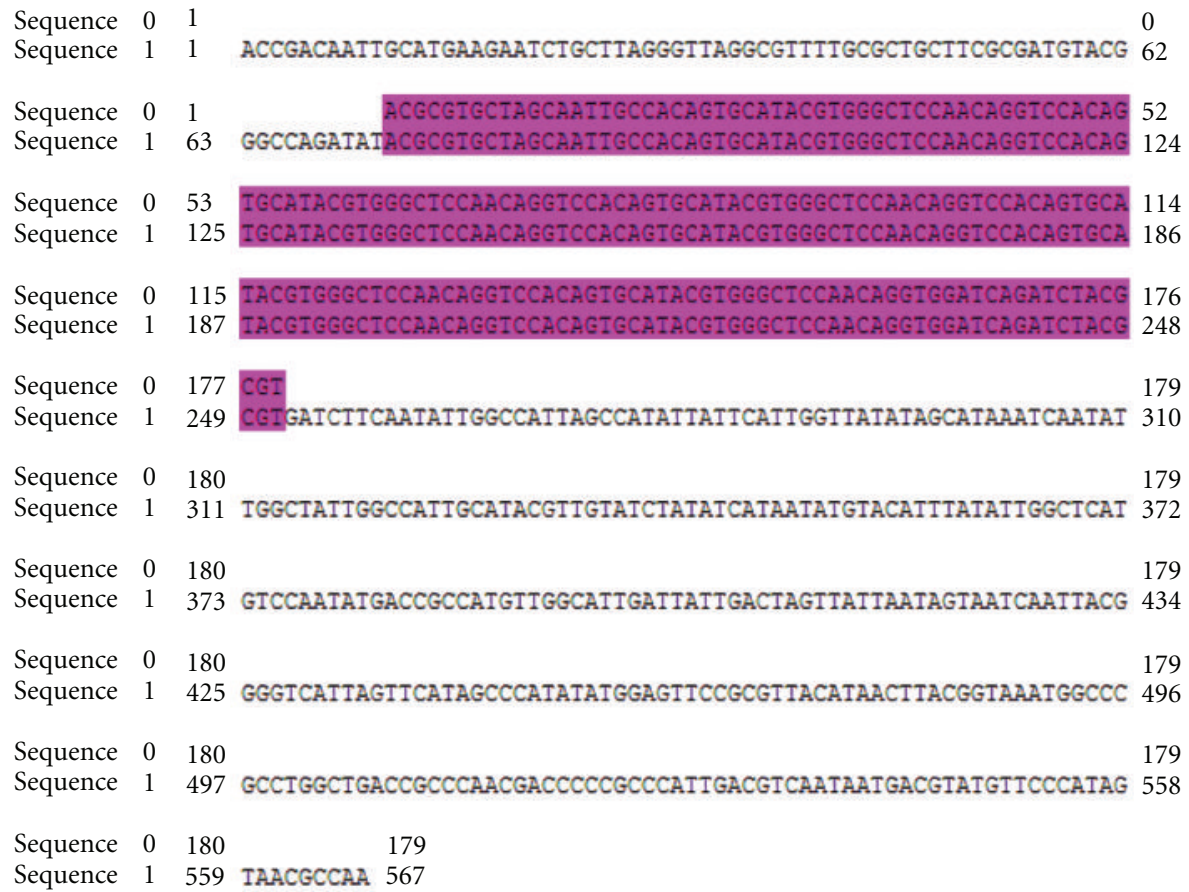

FIGURE 6: Sequencing and alignment of pCDNA3.1-5HRE-Egr1-EGFP (sequence 0: 5HRE, sequence 1: pCDNA3.1-5HRE-Egr1p-EGFP with ab1 reverse complementary sequences; software: Dnassit 2.0).

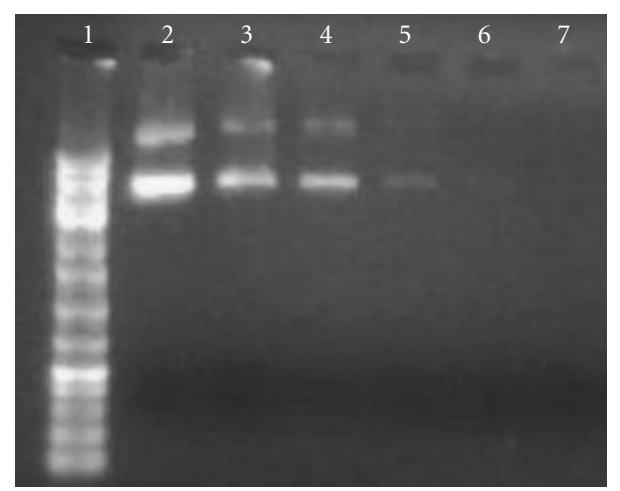

FIGURE 7: Image of agarose gel electrophoresis of pHRE-Egr1-EGFP, free and mixed with PEI-MZF-NPs at different mass ratios (PEIMZF-NPs: pHRE-Egr1-EGFP) (lane 1: Mark; lane 2: $0: 1$; lanes 3 to 5: 1; lane 4: $10: 1$; lane 5: 20:1; lane 6: $40: 1$; lane 7: $80: 1$ ).

2.7.3. MTT Assay for DNA-PEI-MZF-NPs Cytotoxicity. Bel7402 cells, transfected by the above three methods, were subcultured in 96-well plates $\left(2 \times 10^{3} /\right.$ well $)$ for $72 \mathrm{~h}$ in air $5 \%$ $\mathrm{CO}_{2}$ at $37^{\circ} \mathrm{C}$, respectively. The same number of cells seeded without transfection were taken as the negative control. Then $20 \mu \mathrm{L}$ ( $5 \mathrm{~g} /$ liter) of MTT was added to the cells in every well and incubated for $4 \mathrm{~h}$ at $37^{\circ} \mathrm{C}$. The culture medium was replaced by $150 \mu \mathrm{L}$ of DMSO and vibrated for $10 \mathrm{~min}$. Then the optical density (OD) values were measured at a wavelength of $493 \mathrm{~nm}$ using a microplate reader (Multiskan MK3-353, USA). The cell survival ratio was counted with the following formula: survival ratio $(\%)=(\mathrm{OD}$ of the

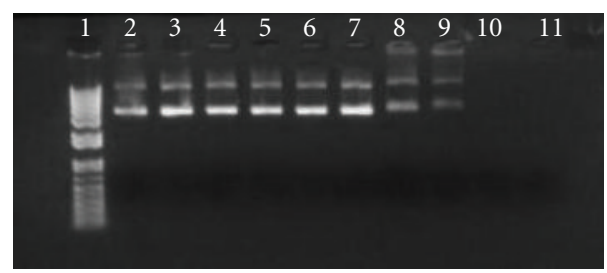

Figure 8: DNase-I resistance of pHRE-Egr1-EGFP-PEI-MZF-NPs (lane 1: Mark; lane 2: DNA-PEI-MZF-NPs with DNase-I for $10 \mathrm{~min}$; lane 3: DNA-PEI-MZF-NPs with DNase-I for $20 \mathrm{~min}$; lane 4: DNAPEI-MZF-NPs with DNase-I for $30 \mathrm{~min}$; lane 5: DNA -PEI-MZFNPs with DNase-I for $40 \mathrm{~min}$; lane 6: DNA -PEI-MZF-NPs with DNase-I for $60 \mathrm{~min}$; lane 7: DNA without DNase-I; lane 8: DNA with DNase-I for $10 \mathrm{~min}$; lane 9: DNA with DNase-I for $20 \mathrm{~min}$; lane 10: DNA with DNase-I for $30 \mathrm{~min}$; lane 11: DNA with DNase-I for $40 \mathrm{~min}$ ).

experimental group $) /(\mathrm{OD}$ of the negative control group $) \times$ $100 \%$.

2.8. Statistical Analysis. Values were expressed as mean \pm SD. The data were analyzed with the SPSS 14.0 program, with $P<0.05$ taken as the criterion for statistical significance.

\section{Results and Discussion}

3.1. Construction and Identification of pHRE-Egr1-EGFP Plasmid. Recombinant plasmid of pCDNA3.1-Egr1-EGFP was confirmed by PCR amplification and gene sequence analysis, which can be seen from Figure 1 with Egr1 specific banding in lane 2 and lane 4 and Figure 2 with the same sequence as that of Egr1 template. 


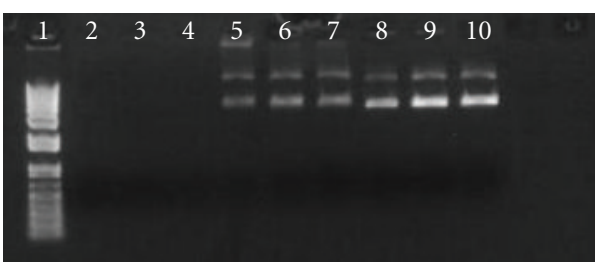

FIgURE 9: In vitro DNA release of PEI-MZF-NPs (lane 1: Mark; lane 2: $1 \mathrm{~h}$; lane 3: $4 \mathrm{~h}$; lane 4: $8 \mathrm{~h}$; lane 5: $12 \mathrm{~h}$; lane 6: $1 \mathrm{~d}$; lane 7: $2 \mathrm{~d}$; lane 8: $3 \mathrm{~d}$; lane 9: $4 \mathrm{~d}$; lane 10: $5 \mathrm{~d}$ ).

If 5HRE fragment was successfully inserted, the fragment length between NdeI and HindII should be about $480 \mathrm{bp}$, because the length between NdeI and HindIII in empty pUC57 vector is about $290 \mathrm{bp}$. As shown in Figure 3 of restriction enzyme digestion and Figure 4 of sequencing, PUC57-5HRE was cloned correctly. Figure 5 was the restriction pattern of pCDNA3.1-5HRE-Egr1-EGFP, and Figure 6 was the HRE sequencing of pCDNA3.1-5HRE-Egr1-EGFP. Theoretically, there was only one BglII restriction site in the original pCDNA3.1-Egr1p-EGFP, if 5HRE was successfully inserted into the plasmid, another BglII restriction site would be brought in, and the fragment length between the two sites of BglII should be about $380 \mathrm{bp}$. Seen from Figure 5, 5HRE has been successfully inserted into pCDNA3.1-Egr1p-EGFP. As shown in Figure 6, DNA sequencing is quite correct. So it can be seen that the eukaryotic recombinant plasmid pCDNA3.1-HRE-Egr1p-EGFP was successfully constructed.

3.2. pHRE-Egr1-EGFP Packaging of PEI-MZF-NPs. Figure 7 represents the results of agarose gel electrophoresis of the composite with different proportion of pHRE-Egr1-EGFP and PEI-MZF-NPs. A clear DNA band could be seen in each lane with $1: 0,1: 5$ and $1: 10$ of DNA and PEI-MZF-NPs, and the band in the lane with $1: 20$ was significantly weakening, and there was no band to be seen in either the lane with $1: 40$ or over. These results suggested that PEI-MZF-NPs could combine all the plasmids in the system at the ratio of $1: 40$ and beyond, and 1:40 was the optimal proportion.

3.3. Nuclease Resistance of the Composite. When exposed to DNase I for 60 minutes, no change in strip brightness could be observed in the case of compound nanoparticles, whereas, with the digestion time extended, gradual degradation of naked DNA was apparent, and almost all was digested 40 minutes later (Figure 8). These results indicated that the DNA was stably incorporated within the nanoparticle matrix and therefore effectively protected from nuclease degradation. The reason for this protection against enzymatic digestion may be due to (1) repulsion of $\mathrm{Mg}^{2+}$ ions (which are necessary for the enzymatic reaction) by the amino groups, (2) a hindered access of the enzymes to the DNA that was immobilized on the nanoparticle surface, or (3) both (1) and (2) [36].

3.4. PEI-MZF-NPs Release. To be a candidate as a DNA carrier, the plasmid should be released from the particles in

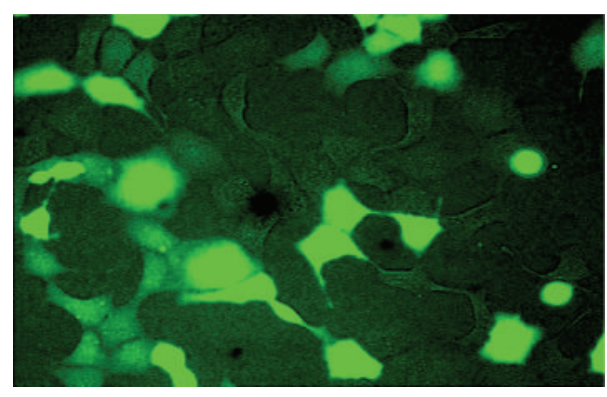

(a)

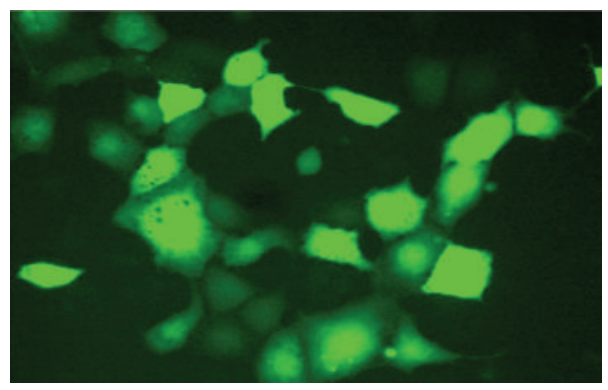

(b)

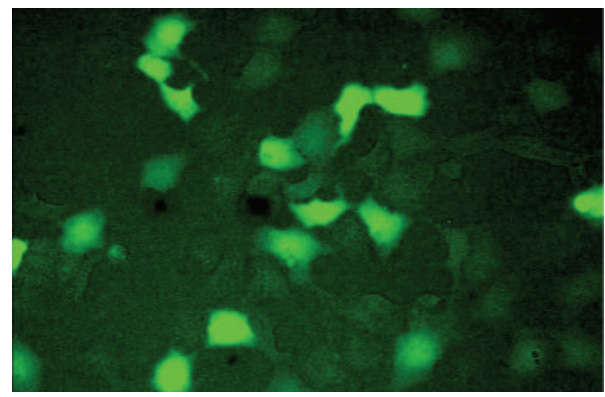

(c)

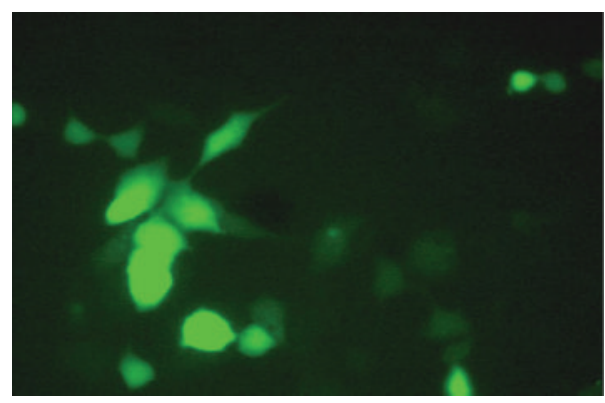

(d)

Figure 10: Bel-7402 cells transfected with pHRE-Egr1-EGFP delivered with PEI-MZF-NPs, electroporation, and liposome and induced by double hypoxia-radiation or radiation alone. The observed green cellular fluorescence is from the expressed EGFP, which has been confirmed by fluorescence microscopy ((a) magnetic nanoparticle group induced by hypoxia-radiation; (b) electroporation group induced by hypoxia radiation; (c), liposome group induced by radiation-hypoxia; (d) magnetic nanoparticle group induced by radiation alone). 


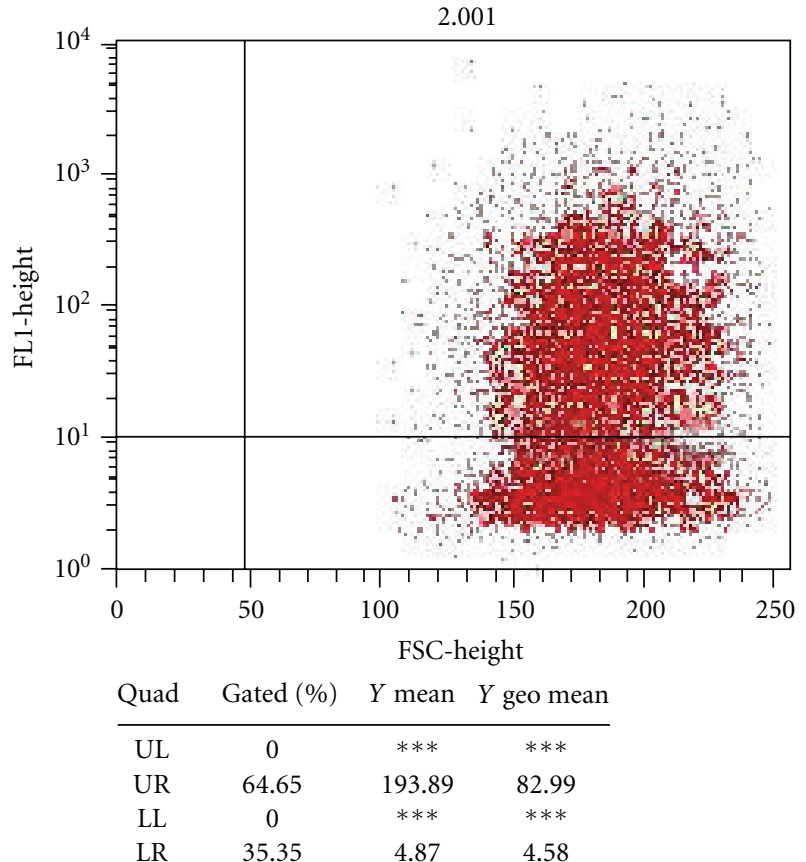

(a)

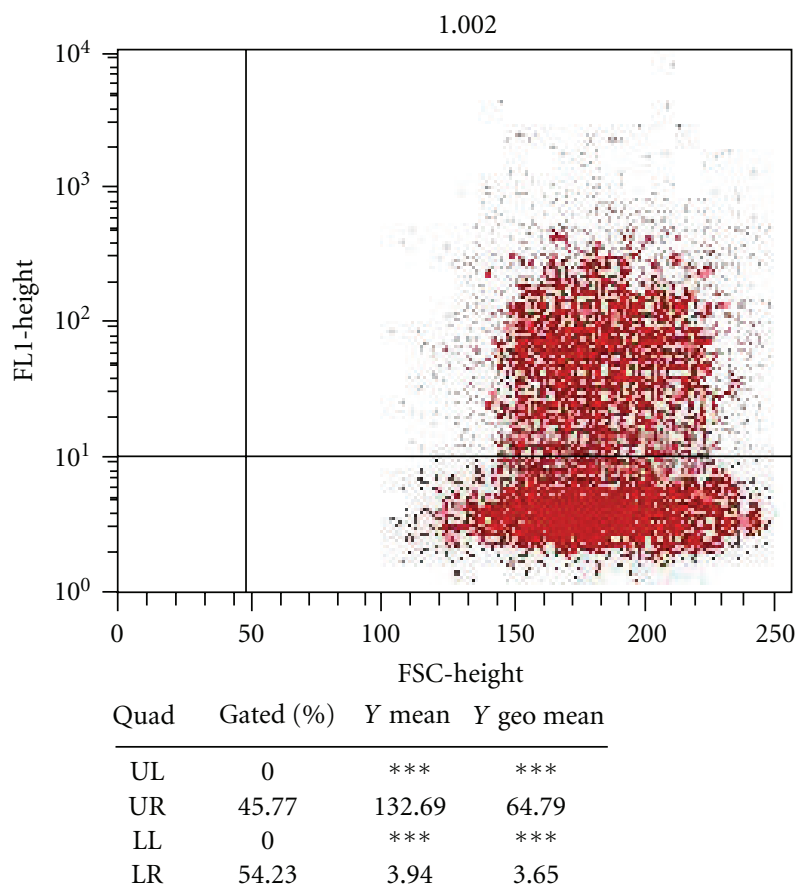

(c)

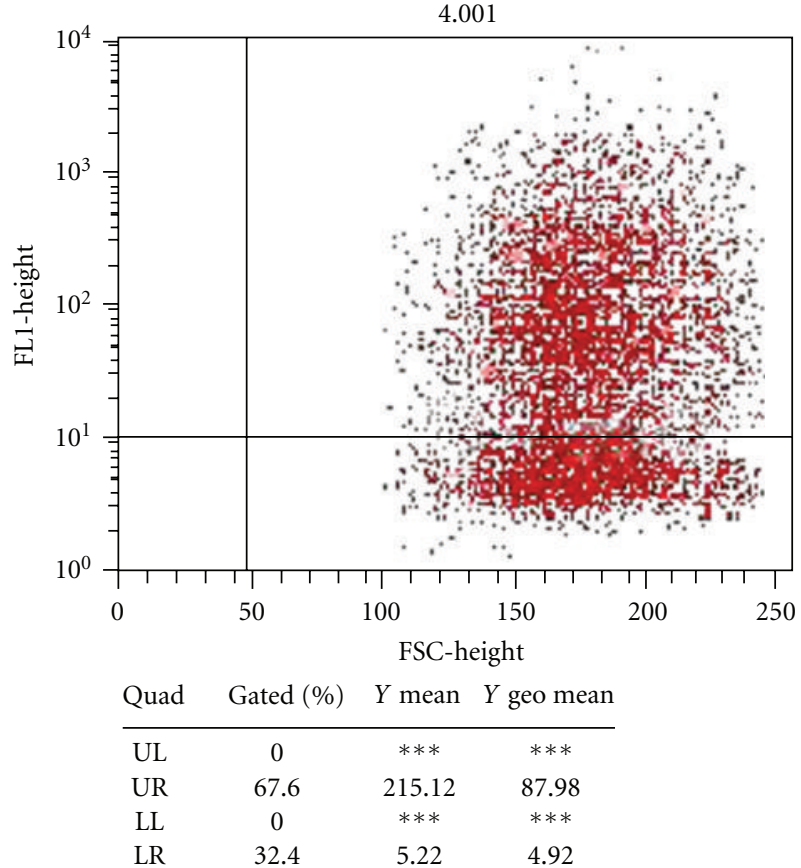

(b)

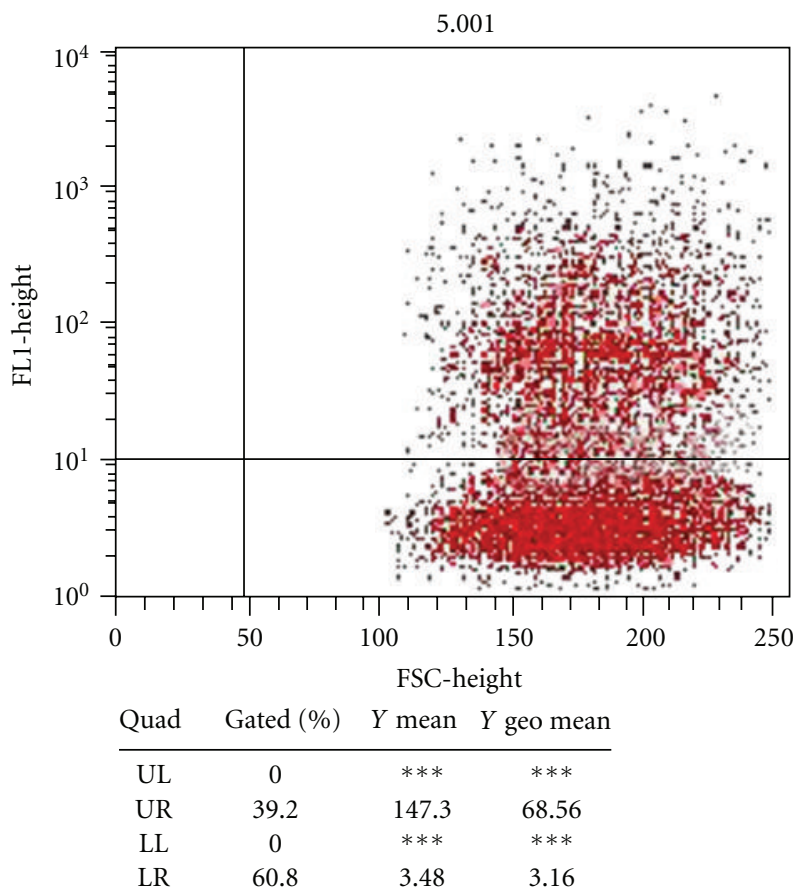

(d)

FIGURE 11: Flow cytometry showed the expression of EGFP in Bel-7402 cells with pHRE-Egr1-EGFP delivered by PEI-MZF-NPs, electroporation, and liposome and induced by double hypoxia radiation or radiation alone ((a) magnetic nanoparticle group induced by hypoxia-radiation; (b) electroporation group induced by hypoxia radiation; (c) liposome group induced by radiation hypoxia; (d) magnetic nanoparticle group induced by radiation alone). 
TABle 1: Comparison of toxicity on Bel-7402 cells transfected by means of PEI-MZF-NPs, electric perforation, and liposome over a period of $72 \mathrm{~h}(\bar{x} \pm s, n=5)$.

\begin{tabular}{lcc}
\hline Group & $\begin{array}{c}\text { Optical density } \\
(\text { OD })\end{array}$ & $\begin{array}{c}\text { Relative survival } \\
\text { rate }(\%)\end{array}$ \\
\hline Negative control group & $0.599 \pm 0.012$ & \\
PEI-MZF-NPS group & $0.581 \pm 0.009^{\mathrm{b}, \mathrm{c}}$ & 97.1 \\
Electricity perforation group & $0.394 \pm 0.009^{\mathrm{a}}$ & 65.8 \\
Liposomes group & $0.249 \pm 0.073^{\mathrm{a}}$ & 41.5 \\
\hline
\end{tabular}

${ }^{a} P<0.001$ versus negative control group; ${ }^{b} P<0.001$ versus electricity perforation group. ${ }^{c} P<0.001$ versus liposomes group.

order to allow its expression in cells. The release increased over the first $3 \mathrm{~d}$, showing gradual increase in brightness of electrophoretic bands, reaching the maximum on the 3rd day and then leveled off (Figure 9). It was confirmed, thus, that besides good DNA-binding ability and protection from degradation, PEI-MZF-NPs have a good DNA release ability in appropriate conditions.

3.5. Carrying Efficiency and Cytotoxicity In Vitro. Under fluorescence microscope, green fluorescence in the three transfected groups was visible, and the cells with green fluorescence in electroporation group were more and brighter than those in the other two groups, and the PEI-MZF-NPs group came next, the liposome group the worst, and no green fluorescence in the untransfected group (Figure 10).

Tested by flow cytometry (Figure 11), transfection ratio and mean fluorescence intensity of magnetic nanoparticle group were $64.65 \%$ and 193.89 , respectively, slightly lower than $67.60 \%$ and 215.12 of electroporation group, but obviously higher than $45.77 \%$ and 132.69 of liposome group. Moreover, in the magnetic nanoparticle groups, transfection ratio and fluorescence intensity induced by radiation and hypoxia both were significantly higher than $48.94 \%$ and 169.15 induced by radiation alone, which was consistent with the results observed by fluorescence microscope.

MTT assay is commonly used as a cell proliferation assay. Bel 7402 cells transfected with pHRE-Egr1-EGFP by PEI-MZF-NPs, liposome, electroporation, respectively, were incubated over a period of $5 \mathrm{~d}$, and the cell viability was determined as described above by MTT. As shown in Table 1, optical density (OD) of the negative control was close to that of PEI-MZF-NPs group $(P>0.05)$, without statistically significant differences, but significantly higher than that of electroporation group $(P<0.001)$ and liposome group $(P<0.001)$, with statistically significant differences. The relative survival rate $(97.1 \%)$ of cells in the group transfected by PEI-MZF-NPs was significantly higher than that in electroporation group $(65.8 \%)$ and liposome group $(41.5 \%)$, with electroporation group lower and liposome group lowest, indicating that transfection by electroporation and lipofection method equally has much toxicity on Bel7402 cells, whereas almost no toxicity was detected by means of PEI-MZF-NPs.

\section{Conclusions}

In this study, we developed pHRE-Egr1-EGFP/PEI-MZFNPs composite nano-system in which pHRE-Egr1-EGFP, eukaryotic gene expression plasmid, was constructed by combining radiation promoter Egr 1 with HRE, forming anoxia radiation double sensitive promoter of HRE/Egr1 to activate reporter gene EGFP expression. MZF-NPs, obtained by coprecipitation method, were coated with PEI. The in vitro experimental results demonstrated that PEI-MZF-NPs have good DNA-binding ability, protection ability, release ability, little toxicity, and high transfection efficiency on Bel-7402 cells, obviously superior to those of the liposome and electricity perforation. Moreover, the expression level of EGFP gene induced by anoxia and radiation was significantly higher than that of single radiation activation. It is therefore concluded that HRE/Egrl can induce and improve target gene expression efficiency in cancer anoxic microenvironment, and that PEI-MZF-NPs can be used as a novel non-viral gene vector which offers a viable approach to the mediated radiation-gene therapy of cancer.

\section{Acknowledgments}

The authors extend their sincere thanks to the financial support from National Natural Science Foundation of China (30770584 and 81171452), National Hi-tech Research and Development Program of China (863 Project, 2007AA03Z356), and Natural Science Foundation of Jiangsu, China (BK2010357).

\section{References}

[1] M. Hingorani, C. L. White, A. Merron et al., "Inhibition of repair of radiation-induced DNA damage enhances gene expression from replication-defective adenoviral vectors," Cancer Research, vol. 68, no. 23, pp. 9771-9778, 2008.

[2] H. Tsurushima, X. Yuan, L. E. Dillehay, and K. W. Leong, "Radiation-inducible caspase-8 gene therapy for malignant brain tumors," International Journal of Radiation Oncology Biology Physics, vol. 71, no. 2, pp. 517-525, 2008.

[3] X. J. Xu, L. H. Ding, L. X. Wang et al., "Construction of human Egr-1 promoter and its response to ionizing radiation in tumor cells," Xi Bao yu Fen zi Mian yi Xue za Zhi, vol. 25, no. 11, pp. 973-975, 2009.

[4] G. L. Semenza, "Targeting HIF-1 for cancer therapy," Nature Reviews Cancer, vol. 3, no. 10, pp. 721-732, 2003.

[5] G. Bartholomeusz, P. Cherukuri, J. Kingston et al., "In vivo therapeutic silencing of hypoxia-inducible factor 1 alpha (HIF- $1 \alpha$ ) using single-walled carbon nanotubes noncovalently coated with siRNA," Nano Research, vol. 2, no. 4, pp. 279-291, 2009.

[6] A. Rapisarda, M. Hollingshead, B. Uranchimeg et al., "Increased antitumor activity of bevacizumab in combination with hypoxia inducible factor-1 inhibition," Molecular Cancer Therapeutics, vol. 8, no. 7, pp. 1867-1877, 2009.

[7] L. Marignol, R. Foley, T. D. Southgate, M. Coffey, D. Hollywood, and M. Lawler, "Hypoxia response element-driven cytosine deaminase/5-fluorocytosine gene therapy system: a highly effective approach to overcome the dynamics of tumour hypoxia and enhance the radiosensitivity of prostate cancer 
cells in vitro," Journal of Gene Medicine, vol. 11, no. 2, pp. 169 179, 2009.

[8] T. Shibata, A. J. Giaccia, and J. M. Brown, "Development of a hypoxia-responsive vector for tumor-specific gene therapy," Gene Therapy, vol. 7, no. 6, pp. 493-498, 2000.

[9] B. Kealy, A. Liew, J. M. McMahon et al., "Comparison of viral and nonviral vectors for gene transfer to human endothelial progenitor cells," Tissue Engineering-Part C, vol. 15, no. 2, pp. 223-231, 2009.

[10] H. Akita and H. Harashima, "Nonviral gene delivery," Contributions to Nephrology, vol. 159, pp. 13-29, 2008.

[11] Y. Kaneda and Y. Tabata, "Non-viral vectors for cancer therapy," Cancer Science, vol. 97, no. 5, pp. 348-354, 2006.

[12] E. Cohen-Sela, M. Chorny, D. Gutman et al., "Characterization of monocytes-targeted nanocarriers biodistribution in leukocytes in ex-vivo and in-vivo models," Nano Biomedicine and Engineering, vol. 2, no. 2, pp. 91-99, 2010.

[13] B. Pan, D. Cui, Y. Sheng et al., "Dendrimer-modified magnetic nanoparticles enhance efficiency of gene delivery system," Cancer Research, vol. 67, no. 17, pp. 8156-8163, 2007.

[14] C. Liu, "Research and development of nanopharmaceuticals in China," Nano Biomedicine and Engineering, vol. 1, no. 1, pp. 1-12, 2009.

[15] E. Anton, K. Swetha, W. Thomas et al., "Dextran-based nanocarriers as efficient media delivery vehicles to cell production bioreactors," Nano Biomedicine and Engineering, vol. 2, no. 2, pp. 126-132, 2010.

[16] D. Cui, Y. Han, Z. Li et al., "Fluorescent magnetic nanoprobes for in vivo targeted imaging and hyperthermia therapy of prostate cancer," Nano Biomedicine and Engineering, vol. 1, no. 1, pp. 61-74, 2009.

[17] S. Chen, Y. Ji, Q. Lian et al., "Gold nanorods coated with multilayer polyelectrolyte as intracellular delivery vector of antisense oligonucleotides," Nano Biomedicine and Engineering, vol. 2, no. 1, pp. 15-23, 2010.

[18] P. Huang, Z. Li, and J. Lin, "Photosensitizer-conjugated magnetic nanoparticles for in vivo simultaneous magnetofluorescent imaging and targeting therapy," Biomaterials, vol. 32, pp. 3447-3458, 2011.

[19] G. Gao, P. Huang, Y. Zhang, K. Wang, W. Qin, and D. Cui, "Gram scale synthesis of superparamagnetic $\mathrm{Fe}_{3} \mathrm{O}_{4}$ nanoparticles and fluid via a facile solvothermal route," vol. 13, no. 6, pp. 1782-1785, 2011.

[20] J. Panyam and V. Labhasetwar, "Biodegradable nanoparticles for drug and gene delivery to cells and tissue," Advanced Drug Delivery Reviews, vol. 55, no. 3, pp. 329-347, 2003.

[21] G. Bhakta, S. Mitra, A. Maitra et al., "DNA encapsulated magnesium and manganous phosphate nanoparticles: potential non-viral vectors for gene delivery," Biomaterials, vol. 26, no. 14, pp. 2157-2163, 2005.

[22] S. Liang, Y. Wang, J. Yu, C. Zhang, J. Xia, and D. Yin, "Surface modified superparamagnetic iron oxide nanoparticles: as a new carrier for bio-magnetically targeted therapy," Journal of Materials Science, vol. 18, no. 12, pp. 2297-2302, 2007.

[23] S. Mansouri, P. Lavigne, K. Corsi, M. Benderdour, E. Beaumont, and J. C. Fernandes, "Chitosan-DNA nanoparticles as non-viral vectors in gene therapy: strategies to improve transfection efficacy," European Journal of Pharmaceutics and Biopharmaceutics, vol. 57, no. 1, pp. 1-8, 2004.

[24] V. Vijayanathan, T. Thomas, and T. J. Thomas, "DNA nanoparticles and development of DNA delivery vehicles for gene therapy," Biochemistry, vol. 41, no. 48, pp. 14085-14094, 2002.
[25] J. M. Wang, B. L. Xiao, J. W. Zheng, H. B. Chen, and S. Q. Zou, "Effect of targeted magnetic nanoparticles containing 5 -FU on expression of bcl-2, bax and caspase 3 in nude mice with transplanted human liver cancer," World Journal of Gastroenterology, vol. 13, no. 23, pp. 3171-3175, 2007.

[26] X. Wang, B. Yu, Y. Wu, R. J. Lee, and L. J. Lee, "Efficient downregulation of CDK4 by novel lipid nanoparticle-mediated siRNA delivery," Anticancer Research, vol. 31, no. 5, pp. 16191626, 2011.

[27] Y. Liu, T. Wang, F. He et al., "An efficient calcium phosphate nanoparticle-based nonviral vector for gene delivery," International Journal of Nanomedicine, vol. 6, pp. 721-727, 2011.

[28] M. B. Jesus, C. V. Ferreira, E. Paula et al., "Design of solid lipid nanoparticles for gene delivery into prostate cancer," Journal of Controlled Release, vol. 148, no. 1, pp. e89-e90, 2010.

[29] D. Wang, Y. Lin, L. X. Gu et al., "Study on Properties of nanosized antimony doped tin oxide suspension," China Powder Science and Technology, vol. 1, pp. 10-13, 2004.

[30] F. Gao, B. F. Pan, W. M. Zheng, L. M. Ao, and H. C. Gu, "Study of streptavidin coated onto PAMAM dendrimer modified magnetite nanoparticles," Journal of Magnetism and Magnetic Materials, vol. 293, no. 1, pp. 48-54, 2005.

[31] R. Goyal, R. Bansal, S. Tyagi, Y. Shukla, P. Kumar, and K. C. Gupta, "1,4-Butanediol diglycidyl ether (BDE)-crosslinked PEI-g-imidazole nanoparticles as nucleic acid-carriers in vitro and in vivo," Molecular BioSystems, vol. 7, no. 6, pp. 20552065, 2011.

[32] Q. S. Tang, D. S. Zhang, X. M. Cong, M. L. Wan, and L. Q. Jin, "Using thermal energy produced by irradiation of Mn-Zn ferrite magnetic nanoparticles (MZF-NPs) for heat-inducible gene expression," Biomaterials, vol. 29, no. 17, pp. 2673-2679, 2008.

[33] A. Q. Zheng, X. R. Song, J. M. Yu et al., "Construction of a chimeric promoter HRE/CarG and its regulation of hypoxia and radition on liver cancer cell," Chinese Journal of Cancer Biotherapy, vol. 12, no. 1, pp. 69-71, 2005.

[34] X. Cong, D. Zhang, Q. Tang, N. Gu, S. Zhao, and J. Zhang, "Biocompatibility of $\mathrm{Mn}_{0.5} \mathrm{Zn}_{0.5} \mathrm{Fe}_{2} \mathrm{O}_{4}$ nanoparticles used in tumor hyperthermia," Journal of Southeast University (National Science Education), vol. 3, pp. 23-27, 2007.

[35] Q. S. Tang, D. S. Zhang, and N. Gu, "Synthesis and in vitro study of PEI-coated MneZn ferrite e a novel gene vector," Journal of Functional Materials, vol. 38, Article ID 1268e72, 2007.

[36] O. Bayguinov, B. Hagen, and K. M. Sanders, "Substance P modulates localized calcium transients and membrane current responses in murine colonic myocytes," British Journal of Pharmacology, vol. 138, no. 7, pp. 1233-1243, 2003. 

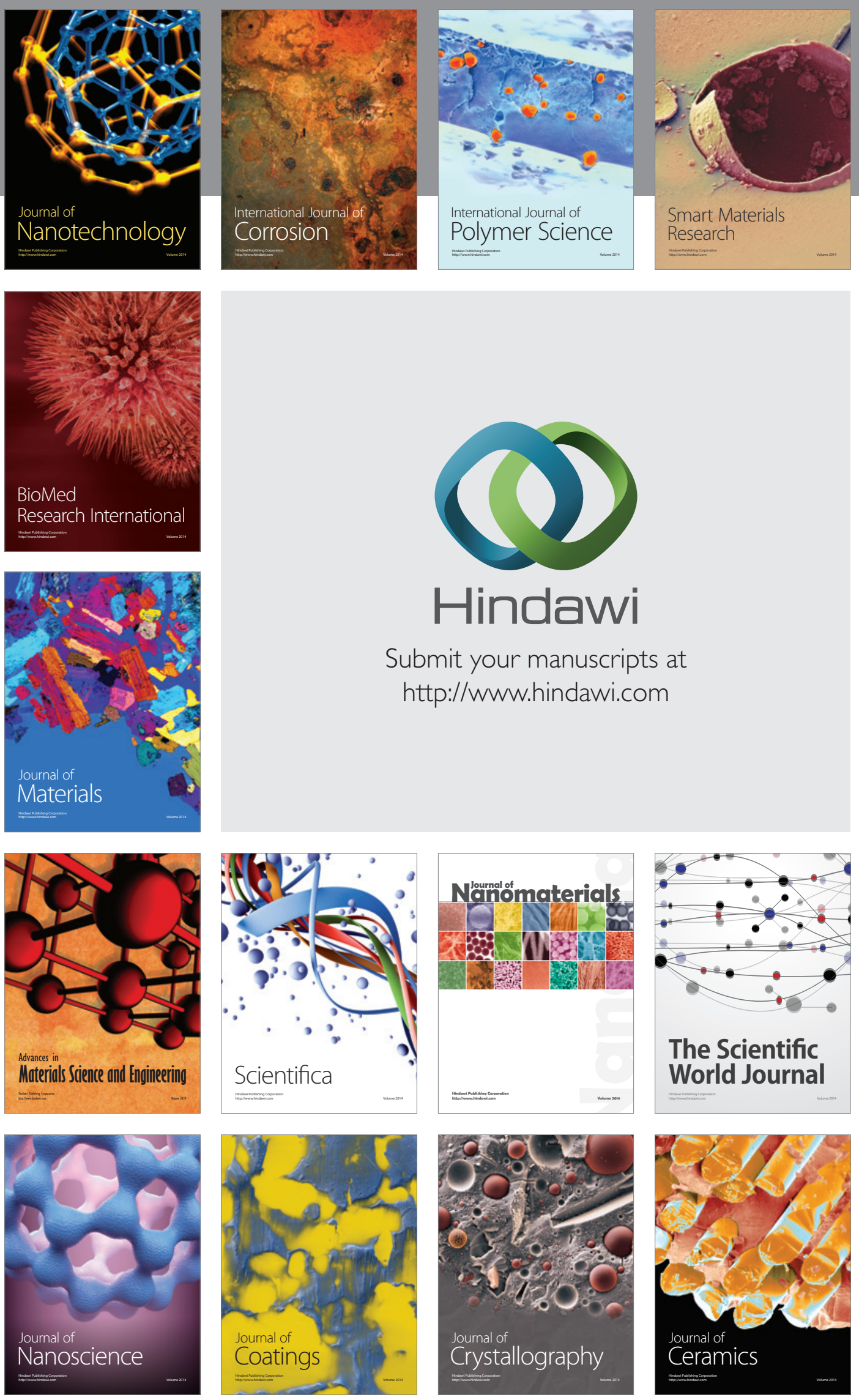

The Scientific World Journal

Submit your manuscripts at

http://www.hindawi.com

\section{World Journal}

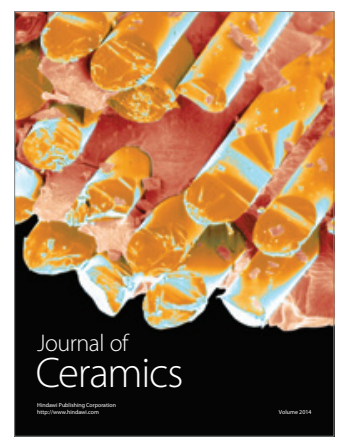

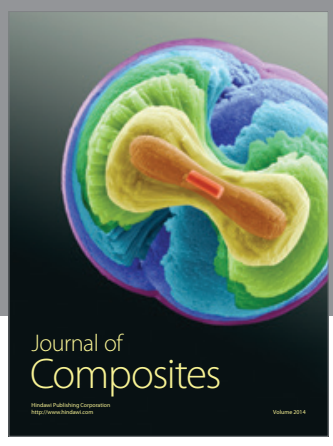
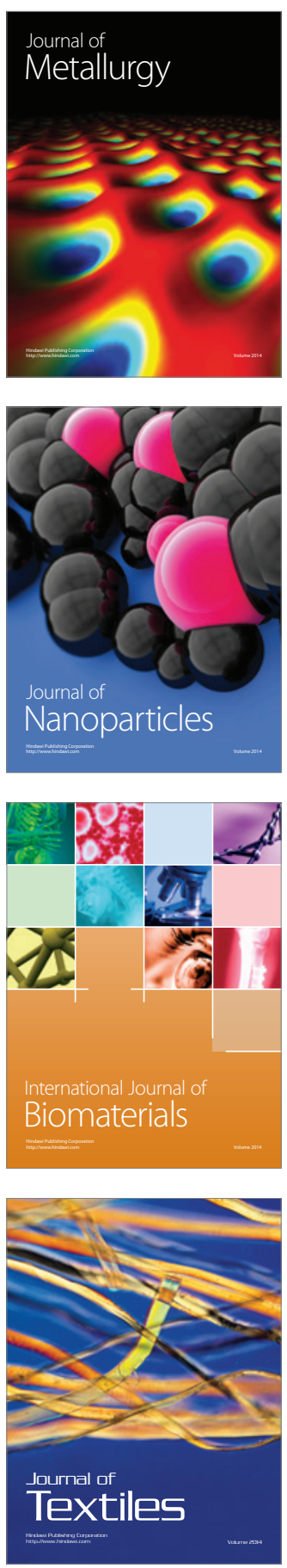